\title{
Mitos y realidades en las prácticas de MU MICONE (Myths and realities in MA MICONE)
}

\author{
Carmen Valero Garcés \\ Carmen.valero@uah.es \\ Departamento de Filología Moderna \\ Universidad de Alcalá \\ Alcalá de Henares, Madrid, España
}

\begin{abstract}
Resumen-El plan de estudios del Máster Universitario (MU) en Interpretación de Conferencias orientado a los Negocios (MICONE) de la Universidad de Alcalá incluye la asignatura de Prácticas (6 ECTS) en empresas u organismos previa firma de un convenio educativo entre éstas y la Universidad. Las restricciones de movilidad y presencialidad por la pandemia del COVID 19, así como la anulación de congresos, ferias, o reuniones de negocios hizo necesaria la búsqueda de otras alternativas para que los alumnos del curso 20202021 pudiesen completar sus estudios. Esta propuesta presenta la experiencia llevada a cabo con los alumnos de interpretación del Máster MICONE y grupo de investigación GIECO para la realización de sus prácticas y la evaluación de los resultados.
\end{abstract}

\section{Palabras clave: prácticas curriculares, interpretación, online.}

Abstract- MA in Conference Interpreting Business Oriented (MICONE) at the University of Alcalá includes the compulsory subject Internships (6 ECTS) in companies or organizations after signing an educational agreement. The restrictions on mobility and attendance due to the COVID 19 pandemic, as well as the cancellation of congresses, fairs, or business meetings made it necessary to look for other alternatives so that students in the 2020-2021 academic year could complete their studies. This proposal presents the experience carried out with the MA MICONE and the research group GIECO to complete the students' internships, and the evaluation of the results.

\section{Keywords: internship, interpreting, online}

\section{INTRODUCCIÓN}

Las prácticas en empresas forman parte casi inexcusable de la formación de postgrado dado el interés en preparar a los alumnos en su incorporación al mundo laboral. Son varios los estudios que analizan, generalmente de un modo cualitativo, las evidencias sobre los beneficios de las prácticas en empresas (PAE) y en diferentes ámbitos. Estas investigaciones tienden a estar basadas en información cualitativa (Silva et al., 2010, Matthew, Taylor \& Ellis, 2012; Callanan \& Benzing, 2007; Gault et al., 2000; Montoro-Sánchez et al., 2012). En cuanto a estudios cuantitativos, como apunta Di Maglio et al (2019), a pesar de ser implementadas masivamente por las universidades españolas, son más bien escasos a la hora de evaluar su eficacia para la inserción laboral. Las firma de convenios con la empresas y los protocolos o normas que cada centro educativo establece es otro de los puntos clave de estas prácticas que dejaremos para otra ocasión. La pandemia cambió este panorama aún más y hubo que buscar otras alternativas, que pueden convertirse en un instrumento que favorezca la inserción laboral de los estudiantes.

El Máster Universitario en Interpretación de Conferencias orientado a los Negocios (MICONE) de la Universidad de Alcalá es un máster de 60 ECTS y se ofrece en dos combinaciones lingüísticas o especialidades: inglés-español y chino-español. MICONE se inició en el curso 2019- 2020. Los alumnos reciben formación, entre otras, en interpretación bilateral, consecutiva y simultánea en los idiomas de su combinación lingüística y se especializan en el ámbito de los negocios y comercio internacional. Según el plan de estudios es necesario realizar una práctica externa de 6 ECTS en empresas $\mathrm{u}$ organismos públicos o privados. La asignatura permite conocer el funcionamiento de las empresas o instituciones, sus métodos y técnicas de trabajo, como complemento práctico de los estudios de interpretación de conferencias y los negocios y conocer la especificidad de la interpretación de conferencias (IC) in situ. Las competencias generales que adquieren a lo largo del curso son, entre otras, aplicar la capacidad para la planificación y gestión del tiempo; aplicar los conocimientos adquiridos y su capacidad de resolución de problemas en el lugar de la práctica; saber integrar conocimientos y enfrentarse a la complejidad de un encargo de interpretación y desarrollar habilidades de autoaprendizaje.

Junto a estas competencias otras más específicas relacionadas con su tema de estudio que la realización de las prácticas lleva consigo son: conocimiento del mercado profesional de la IC y las características laborales y el desarrollo de la profesión de intérprete; comprender la jerga profesional del ámbito en el que se interpreta; aplicar con eficacia habilidades para las relaciones interpersonales y con los clientes; saber trabajar en equipo y en un contexto internacional e interdisciplinar y ampliar conocimientos sobre tipos y géneros textuales típicos de la interpretación simultánea así como de discursos especializados.

Llevado a la práctica, y como parte del encargo de traducción, entre las labores que los alumnos realizan durante las prácticas se hallan las siguientes: proporcionar asistencia en reuniones o conferencias como enlace entre representantes hablantes de chino o inglés y español; interpretar las grabaciones de conferencias o vídeos corporativos a los idiomas chino, inglés y español con el objetivo de que su contenido sea 
accesible en más idiomas;- investigar la comercialización de productos similares o campañas publicitarias en chino, inglés o español para conocer las estrategias de la competencia o en otros países y adaptarlo a las necesidades de la empresa; elaborar / traducir / adaptar notas de prensa sobre sus productos/ servicios para un público especializado o general (del español al inglés o chino); adaptar al inglés o chino los productos o servicios que ofrece la empresa.

Las prácticas se desarrollan como se ha indicado en centros o empresas públicas o privadas con las que la universidad tiene firmado un Convenio de Prácticas. Una vez firmado el convenio la empresa realiza una oferta de prácticas. Las ofertas se ponen a disposición del alumnado en la asignatura Prácticas en Empresas e Instituciones de Aula Virtual durante un periodo de tiempo limitado. Los alumnos solicitan las ofertas enviando su currículo y una carta de motivación a través de Aula Virtual a la Coordinación del Programa de Prácticas. La asignación se realiza por parte de la Coordinación tras examinar la documentación entregada y consultar el expediente académico de los alumnos solicitantes. Una vez finalizado el plazo de publicación de la oferta se produce la asignación. La solicitud de una oferta supone un compromiso. En cuanto a la evaluación y seguimiento de la práctica cada alumno tiene asignado un tutor de práctica en la empresa o centro designado para la realización de la misma y un tutor académico en la universidad. Cada uno de ellos valora aspectos concretos de la práctica en porcentajes establecidos para llegar a la nota final. Y todo se gestiona a través del sitio web para gestionar las prácticas de la Universidad conocido como GIPE (Gestión Integral de Prácticas Externas. Para información más detallada sobre el procedimiento de firma de convenio, coordinación empresauniversidad, función de los tutores, evaluación de las prácticas y valoración de los resultados se recomienda visitar el sitio web del Servicio de Prácticas de la UAH en: https://www.uah.es/es/conoce-la-uah/organizacion-ygobierno/servicios-universitarios/servicio-de-practicas/.

\section{CONTEXTO}

La pandemia del COVID 19 cambió las reglas y hubo que adaptarse. Las restricciones de movilidad y presencialidad en la universidad así como la anulación de congresos, ferias, o reuniones de negocios hizo necesaria la búsqueda de otras alternativas para que los alumnos del curso 2020-2021 pudiesen completar sus estudios. Surge así la experiencia llevada a cabo con los alumnos de interpretación de MU MICONE para la realización de sus prácticas y la evaluación de los resultados con la firma de un convenio de prácticas entre el grupo de investigación GIECO-AGLAYA y el MU MICONE. Se busca así como estrategia la unión de investigación y formación como puerta de acceso al mercado laboral.

GIECO forma parte de un proyecto más amplio que se conoce como AGLAYA. El proyecto forma parte de los Programas de actividades de I+D en el que participan 13 Grupos de Investigación de 7 Universidades, 30 entidades (institutos, asociaciones, fundaciones) y 7 empresas. Uno de los grupos de investigación es el Grupo GIECO de la UAH, primero grupo en España dedicado a la ecocrítica, con campos de investigación muy variados desde las filologías, pero con el interés común de todos en temas medioambientales reflejados en la literatura. Una de las líneas principales de investigación se centra en concienciación, activismo y justicia medioambiental en las humanidades. En consonancia con esta línea de investigación,
GIECO -AGLAYA tenía previsto organizar un seminario sobre mitos. Se establecieron contactos con los coordinadores y se ofreció la posibilidad de que el seminario contase con la interpretación y traducción en inglés y chino por parte de los alumnos del MU MICONE.

\section{DESCRIPCIÓN}

Se aceptó la propuesta por los investigadores del grupo GIECO y se comenzaron los preparativos del $1^{\circ}$ Seminario online sobre Mitos en Comunidades Indígenas. El seminario, como otros que se organizan regularmente en AGLAYA, pretendía tener continuidad en futuras ediciones para ir explorando mitos originarios en diferentes espacios y momentos de la historia. La primera edición con el subtítulo de "Mitos en comunidades de los pueblos originarios americanos 'traducidos' 'interpretados' 'adaptados', se proponía explorar la realidad y el mito de aquellas poblaciones originarias que convivieron con los conquistadores españoles en su avance por tierras americanas y aprendieron su lengua y costumbres.

GIECO lanzó una petición de propuestas para explorar el tema de los mitos en las comunidades originarias de Latinoamérica desde cualquier ángulo y disciplina. Se indicaba que los trabajos aceptados se expondrían de modo virtual en comunicaciones de 15 minutos y se establecían fechas concretas. Se aceptaron seis propuestas con temas diversos y desde distintos países de Sudamérica y España. Siguen los títulos de las ponencias, nombres de los autores y los países de procedencia con el fin de mostrar la variedad de temas y procedencia:

Argentina Rodríguez Rivera, Naví, desde Nicaragua, presentó el tema "El mito de la Cegua".

Bañuelos Aquino, Víctor Manuel, desde México, presentó el tema "De Nuño de Beltrán Guzmán en caballo a Santiago caballero. La interpretación mítica del caballo en la pacificación de la Nueva Galicia".

Camacho Estrada, Sara, también desde México, habló del “El pensamiento andino descrito metafóricamente como interrumpido por el colonialismo".

Lachica, María Cruz, desde Alcalá, presentó el tema "El mito del buen salvaje americano".

Martínez, Fernanda, hondureña que habló desde Hamburgo, presentó el tema "Perros benditos y perros malditos".

Oliva Cruz, Juan Ignacio, desde las Islas Canarias, hablo de "La Malinche traducida y traicionada: una revisión ecofeminista del mito"

En cuanto a MU MICONE, de acuerdo con el Comunicado de la Conferencia de Rectores de las Universidades Públicas Madrileñas (CRUMA), las universidades públicas madrileñas habían planificado el curso 2020-2021 apostando por la máxima presencialidad posible y la situación llevó, en el marco de cumplimiento de las normas sanitarias establecidas por el Gobierno Central y el Gobierno de la Comunidad Autónoma de Madrid, a modificar la actividad docente y realizar adaptaciones para pasar la enseñanza de modo presencial a online, garantizando el derecho del alumno a seguir con la formación a través de aula virtual en la plataforma Blackboard que es la que usa la UAH. Así si algún estudiante o profesor no podía asistir a clase, bien por motivos de restricciones de movilidad en su país/lugar de residencia o bien por cuestiones de 
confinamiento sanitario, a sesiones o actividades presenciales tenía acceso al contenido de la formación en la plataforma en streaming o en remoto ya que se grabaron las clases.

Tal situación supuso cambios importantes en la mayor parte de las asignaturas que pasaron a ofrecerse de modo presencial a virtual en el caso de la UAH utilizando la plataforma virtual de un modo más intensivo que hasta ese momento y añadiendo nuevas aplicaciones que requirieron el aprendizaje de su manejo tanto por parte de los alumnos como de los profesores. En el caso de la interpretación, el paso de la formación presencial a virtual era aún más complicado dado que los alumnos recibían dicha formación en el aula de interpretación con un equipo específico en cabinas de interpretación, necesario sobre todo para la interpretación simultánea - modalidad habitual de uso en congresos y reuniones, y la plataforma Blackboard no permitía el uso de la interpretación simultánea, lo cual suponía un hándicap para los alumnos.

Por otro lado, se anularon o retrasaron el $90 \%$ de los eventos como conferencias, reuniones de trabajo, seminarios, o encuentros internacionales y fue necesario empezar a buscar otras soluciones para hacer posible la comunicación en eventos en los que había personas que hablaban lenguas distintas y era necesario contar con intérpretes. Ello también supuso que las empresas dejaran de acoger a alumnos en prácticas de interpretación o estuviesen dispuestas a firmar un convenio de prácticas dado que no había trabajo.

Ante tal situación fue necesario buscar otros recursos o modos que permitiese a los alumnos seguir formándose para un mercado laboral distinto del que se había previsto y al mismo tiempo era una buena oportunidad para los alumnos el poder experimentar otras formas de llevar a cabo su trabajo.

El profesorado encargado de la formación llevó a cabo un búsqueda de soluciones para la interpretación remota que se estaban dando y tratar de incorpóralas en el curso. Algunas de ellas fueron la introducción de prácticas de interpretación remota, explorar distintas plataformas que permitían la interpretación simultánea, o explorar herramientas electrónicas para la interpretación (en inglés Computer assisted Interpreting, CAI). ). Se practicó con algunos estos recursos y algunas plataformas de varias formas: con los alumnos en las cabinas y los oradores en remoto y con los alumnos y oradores en remoto. De este modo se fue preparando a los alumnos para la realización de sus prácticas en modo remoto y como un paso más hacia su futura incorporación en el mercado laboral, habid cuenta de la situación actual.

GIECO y MICONE firmaron un convenio de prácticas e hicieron una oferta de prácticas para chino e inglés. La solicitaron 8 alumnos- 4 de chino y 4 de inglés-. El resto de los de los alumnos del máster - 12 en total - o bien convalidaron sus prácticas o bien las realizaron en algunas de las pocas empresa que admitieron alumnos para prácticas online, tema que escapa a este artículo

Previo al evento, y una vez recibidos los resúmenes de las ponencias y los ppt o materiales que los ponentes enviaron, los alumnos iniciaron la preparación del encargo de interpretación.

Previo al evento, y una vez recibidos los resúmenes de las ponencias y los ppt o materiales que los ponentes enviaron, los alumnos iniciaron la preparación del encargo de interpretación. El proyecto formativo diseñado incluía dos elementos claves:

a) Competencias que adquiere el estudiante durante su período en prácticas de acuerdo al Plan de estudio y adaptadas a la situación dentro de la oferta GIECO - UAH. Entre las principales competencias que se trabajaron caben destacar las siguientes:

Adquirir la capacidad para la planificación y gestión del tiempo en las tareas propias de interpretación de conferencias (IC).

Desarrollar habilidades de aprendizaje que permitan continuar estudiando de un modo autónomo.

Aplicar los conocimientos adquiridos en entornos nuevos o poco conocidos dentro de contextos más amplios.

Aumentar el conocimiento del mercado profesional de la IC, así como aspectos profesionales y reflexión crítica sobre las características laborales y el desarrollo de la profesión de intérprete.

Ampliar el dominio de tipos y géneros textuales típicos de la interpretación simultánea así como de discursos especializados.

b) Acciones formativas y actividades propuestas, que incluían la preparación y realización de un encargo real de interpretación de conferencias. Dicho encargo conlleva, entre otras, las siguientes acciones:

- Tareas de documentación sobre el tema de la interpretación. lingüísticas.

Desarrollo de glosarios en sus combinaciones

- $\quad$ Elaboración de un documento que describa y/o resuma los materiales y otros recursos utilizados para preparar la interpretación.

Prácticas simuladas sobre los temas del encargo previos a la interpretación real.

Se realizaron grabaciones en archivo audio de los discursos tanto en versión original (español) como de las interpretaciones de los alumnos (inglés o chino) para su posterior evaluación por parte de los tutores.

La última acción consistió, tanto para los alumnos como para los tutores, en cumplimentar las fichas de evaluación de la actividad según los formularios contenidos en la aplicación de GIPE.

Se creó un enlace en la plataforma ZOOM y se difundió por los canales acostumbrados. El evento fue seguido por una media de treinta personas de diferentes partes del mundo, algunos de los cuales dejaron breves mensajes de agradecimiento por los trabajos presentados y la labor de los intérpretes.

\section{RESUltados}

El día del evento los alumnos en prácticas hicieron interpretación simultánea en inglés y en chino en remoto de las ponencias presentadas desde diferentes lugares del mundo. Los alumnos estuvieron en sus cabinas en la universidad para un mayor control por parte de los tutores académicos de prácticas por si surgía algún imprevisto. Se grabaron las presentaciones así como las interpretaciones. Tras el congreso, las interpretaciones fueron evaluadas por los tutores correspondientes siguiendo la rúbrica correspondiente. 
En cuanto a los intérpretes, las tutoras respectivas evaluaron a los alumnos, de manera general, muy positivamente, aunque con algunas diferencias. Destaca, sobre todo, en algunos casos la correcta interpretación expresiones coloquiales que hacen del discurso algo mucho más natural. Subrayan también la buena entonación y cadencia de los intérpretes y resaltan la evolución que han experimentado a lo largo del curso. Se decide incorporar las grabaciones, con el visto bueno de los alumnos, a un repositorio para la práctica de futuros alumnos y como material de uso por el profesorado.

Por parte de los alumnos que actuaron como intérpretes la valoración fue altamente positiva. Además de realizar sus prácticas, fue la primera interpretación que realizaron dirigida al público en general. Sus comentarios revelaron que habían sufrido momento de estrés y pánico, algunos problemas de compresión de términos o de vocabulario específico tanto por el tema como por el país del que hablaban, dificultades para seguir a algún ponente, etc.

Por parte del GIECO-AGLAYA hubo plena satisfacción y se comprometieron a requerir los servicios de nuestros alumnos de MICONE en futuros eventos. Todo el seminario se interpretó al chino y al inglés, permitiendo así que el seminario llegase a un público más amplio.

\section{CONCLUSIONES}

La experiencia de interpretar en directo se recoge dentro de actividades de prácticas del Máster, y les dio la oportunidad a los alumnos de interpretar en un contexto real, familiarizándose también con la realidad actual de la interpretación mediante plataformas virtuales y nuevos recursos para la interpretación asistida por ordenador (CAI- Computer Assisted Interpreting). El éxito del evento y el interés de los alumnos llevo a que se ofreciese esa oportunidad de nuevo a los alumnos en las Jornadas de Empleabilidad que la UAH organizó para los alumnos. Ello les permitió de nuevo enfrentare a interpretar en un entorno real y sobre un tema de importancia para su propio futuro como es conocer el mercado laboral. Las prácticas ayudaron a los estudiantes a desarrollar las competencias requeridas en la asignatura, como competencias de comunicación y de gestión de problemas, el uso de nuevas tecnologías de comunicación e información, competencias que les permitan desarrollar mayores actitudes para la empleabilidad de los estudiantes. Además, generan expectativas más realistas en los estudiantes al enfrentarse por primera vez a un encargo real con público y en distancia. En definitiva, hubo satisfacción tanto de los estudiantes con del grupo GIECO y de los formadores.

La situación específica a la que llevó la pandemia de la COVID 19 propició el desarrollo de actividades formativas de diferente índole cuyas consecuencias están aún por ver. Esta experiencia fue sin duda positiva y permitió cumplir con los requisitos y de la asignatura Prácticas Externas. No obstante, sería deseable poder repetir la experiencia y mejorar algunos puntos como por ejemplo disponer de más tiempo para la preparación del encargo por parte de los alumnos, contar con la colaboración de los ponentes para el envió de materiales que ayuden a los alumnos a conseguir las competencias marcadas, articular mecanismos para facilitar la elaboración de glosarios o practicar con nuevas herramienta $\mathrm{CAI}$ que van surgiendo par la práctica de la IC.

\section{AgRAdECIMIENTOS}

Mi agradecimiento a los participantes en el $1^{\circ}$ Seminario Mitos por colaborar y ayudar a los alumnos de MICONE a formarse y darles la oportunidad de interpretar en directo.

\section{REFERENCIAS}

Callanan, G. \& Benzing, C. (2007).Assessing the role of internships in the career-oriented employment of graduated college students. Education and Training, 46(2), 82-89.

Di Meglio, G.; Barge-Gil, A.; Camiña, E. \& Moreno, L. (2019). The impact of internships on job attainment: an applied analysis of Economics and Business Administration degrees. Educación XX1, 22(2), 235-266, doi: 10.5944/educXX1.22579.

Gault, J., Redington, J., \& Schlager, T. (2000). Undergraduate Business Internships and Career Success: Are They Related? Journal of Marketing Education, 22(1), 45-53.

Matthew. S.M., Taylor, R.M., \& Ellis, R.A. (2012). Relationships between students' experiences of learning in an undergraduate internship programme and new graduates' experiences of professional practice. Higher Education 64 (4), 529-542.

Montoro-Sánchez, M.A., Mora-Valentín, E.M. y Ortiz de Urbina-Criado, M. (2012). Análisis de las competencias adquiridas en los estudios de Dirección de Empresas y su grado de aplicación en las prácticas en empresas. Revista Complutense de Educación, 241, 241-263.

Silva, P., Lopes, B., Costa, M., Seabra, D., Melo, A.I., Brito, E., \& Paiva Dias, G.(2016). Stairway to employment? Internships in higher education. Higher Education, 702, 703-721. 